\title{
Corrosion thickness loss monitoring using high-frequency guided ultrasonic waves
}

Fromme, P., Masserey, B.

P. Fromme, B. Masserey, "Corrosion thickness loss monitoring using highfrequency guided ultrasonic waves," Proc. SPIE 11593, Health Monitoring of Structural and Biological Systems XV, 115931I (22 March 2021); doi: $10.1117 / 12.2582513$

SPIE. Event: SPIE Smart Structures + Nondestructive Evaluation, 2021, Online Only 


\title{
Corrosion thickness loss monitoring using high frequency guided ultrasonic waves
}

\author{
P. Fromme ${ }^{\text {a) }}$, B. Masserey ${ }^{\text {b) }}$ \\ a) Department of Mechanical Engineering, University College London, UK \\ b) Department of Mechanical Engineering, HES-SO University of Applied Sciences and Arts, \\ Fribourg, Switzerland
}

\begin{abstract}
Corrosion thickness loss due to adverse environmental conditions of pipelines and marine structures can cause degradation of structural health. Monitoring in difficult to access areas can be achieved using high frequency guided waves propagating along the structure, selectively excited using standard ultrasonic angle beam transducers with single sided access. Wave propagation and mode interference depends on the thickness of the structure. At the frequency-thickness range of interest, the two fundamental Lamb wave modes are excited with slightly different wavenumbers, leading to a beating effect with energy transfer through the structure thickness. The beating effect depends on the frequency-thickness product and has been found to be very sensitive to small thickness changes. The guided wave propagation and energy transfer were visualized and predicted using 2D Finite Element simulations. Excellent agreement was found to theoretical beatlength predictions from a fit of the recorded variation of guided wave amplitude along the propagation direction. Laboratory experiments were conducted, with steel specimen wall thickness reduced by consecutive milling and using accelerated corrosion. Signal changes due to the wave mode interference were measured and the wall thickness reduction monitored from the amplitude beatlength. Good agreement with the theoretical predictions was achieved, demonstrating the sensitivity for thickness loss monitoring.
\end{abstract}

Keywords: Corrosion, Guided Waves, Wall Thickness Loss, Lamb Waves, Pipelines, Marine Structures

\section{INTRODUCTION}

Wall thickness loss due to corrosion constitutes an important problem for pipelines and marine structures, limiting service life $[1,2]$. Nondestructive ultrasonic measurements have good sensitivity for the quantification of small wall thickness losses due to corrosion [3]. The monitoring of large structures, e.g., pipelines or offshore oil platforms, can be achieved using low frequency guided ultrasonic wave inspection systems $[4,5]$. Low frequency guided wave modes can propagate over long distances and are scattered at defects and features in the structure [6], allowing for the efficient monitoring of large technical structures [7]. As guided wave modes are dispersive, they are sensitive to thickness reduction, which has been employed for corrosion detection $[8,9]$. Guided wave array systems allow for the efficient monitoring of the structural integrity from restricted monitoring locations $[10,11]$.

Operating in the higher frequency-thickness range, guided waves have been employed for non-destructive monitoring [12], achieving good sensitivity for wall thickness loss [13]. Frequency-thickness products of $5 \mathrm{MHz} \mathrm{mm}, 15 \mathrm{MHz} \mathrm{mm}$, and 20 $\mathrm{MHz} \mathrm{mm}$ were respectively employed for corrosion detection in aircraft structures [14], the inspection of plate structures [15], and higher order mode clusters to monitor plates [16]. Higher frequencies result in higher attenuation and more complex guided wave signals, but achieve better sensitivity for the detection of small defects, as wavelengths are comparable to those used in bulk wave ultrasonic testing (UT) [15], e.g., M-Skip [17]. Different guided wave modes and choice of operating point (e.g., $\mathrm{A}_{1}$ Lamb mode, $\mathrm{SH}_{1}$ shear horizontal mode and lower frequency guided waves) were systematically compared [18].

Health Monitoring of Structural and Biological Systems XV, edited by Paul Fromme,

Zhongqing Su, Proc. of SPIE Vol. 11593, 115931l · (c) 2021 SPIE

CCC code: $0277-786 X / 21 / \$ 21 \cdot$ doi: $10.1117 / 12.2582513$

Proc. of SPIE Vol. 11593 115931I-1 


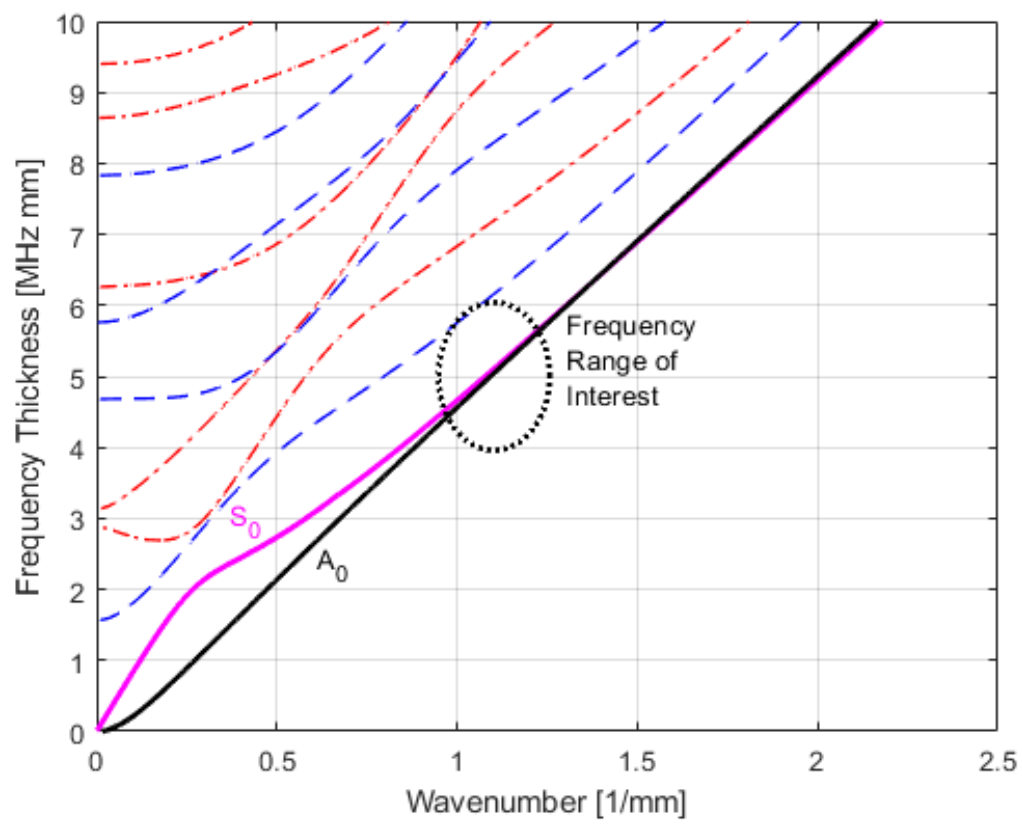

Figure 1. Dispersion diagram for mild steel plate; frequency-thickness product of interest around $5 \mathrm{MHz} \mathrm{mm}$ (center frequency $0.5 \mathrm{MHz}$; approximately $10 \mathrm{~mm}$ thickness) marked.

High frequency guided ultrasonic waves in the frequency-thickness regime around 6-7 MHz mm were used for the detection and localization of defects in multi-layered plate structures [19] and for fatigue crack monitoring [20]. The generated guided waves consisted of a superposition of the fundamental $\mathrm{A}_{0}$ and $\mathrm{S}_{0}$ Lamb modes [21]. Combining time-offlight and frequency analysis, defect location and damaged plate side could be determined [22].

This contribution describes numerical simulations and experiments using high frequency guided ultrasonic waves to monitor thickness reduction in mild steel specimens [2]. The wave propagation and energy transfer across the specimen thickness was visualized using 2D Finite Element (FE) simulations [23] and depends strongly on the difference in wavenumbers according to the frequency-thickness product. This is characterized by the so-called beatlength [24] or beat wavelength [25]. The concept was applied to milled specimens for thickness reduction quantification and to specimens subjected to accelerated corrosion to verify the applicability for wall thinning monitoring.

\section{NUMERICAL SIMULATIONS}

Explicit Finite Element (FE) simulations were conducted using the commercial software ABAQUS to visualize and predict the guided wave propagation and mode interference [23]. A two-dimensional (2D) model of the plate cross-section of $600 \mathrm{~mm}$ length and 9-11 mm thickness was developed. Material properties for mild steel (Young's modulus $200 \mathrm{GPa}$, Poisson's ratio 0.29 , density $7900 \mathrm{~kg} / \mathrm{m}^{3}$ ) were used. The mesh was a Cartesian grid using four-node plane strain elements (CPE4R) with $0.1 \mathrm{~mm}$ element size. This more than fulfilled the relevant numerical discretization criteria with approximately 60 elements per wavelength $(5.8 \mathrm{~mm}$ Rayleigh wavelength at $0.5 \mathrm{MHz}$, see dispersion diagram in Fig. 1), but the fine grid was required to reduce the numerical dispersion to an acceptable level.

Overall simulation time of $0.2 \mathrm{~ms}$ and time step of $10 \mathrm{~ns}$ were used to fulfill the stability criteria and for the waves to propagate the complete plate length. The experimentally observed amplitude reduction was approximated by Rayleigh damping $(\beta=0.5 \mathrm{~ns})$. The excitation signal was a 10 -cycle tone burst $(0.5 \mathrm{MHz}$ center frequency sinusoid in Hanning window). To selectively excite the fundamental $A_{0}$ and $S_{0}$ Lamb wave modes, in-plane and out-of-plane displacements (phase shift of $\pi / 2$ ) of the Rayleigh mode shape at $0.5 \mathrm{MHz}$ were prescribed along a vertical line at the plate end. Out-ofplane displacement signals at $1 \mathrm{~mm}$ spaced monitoring points were recorded along the top and bottom surfaces. 

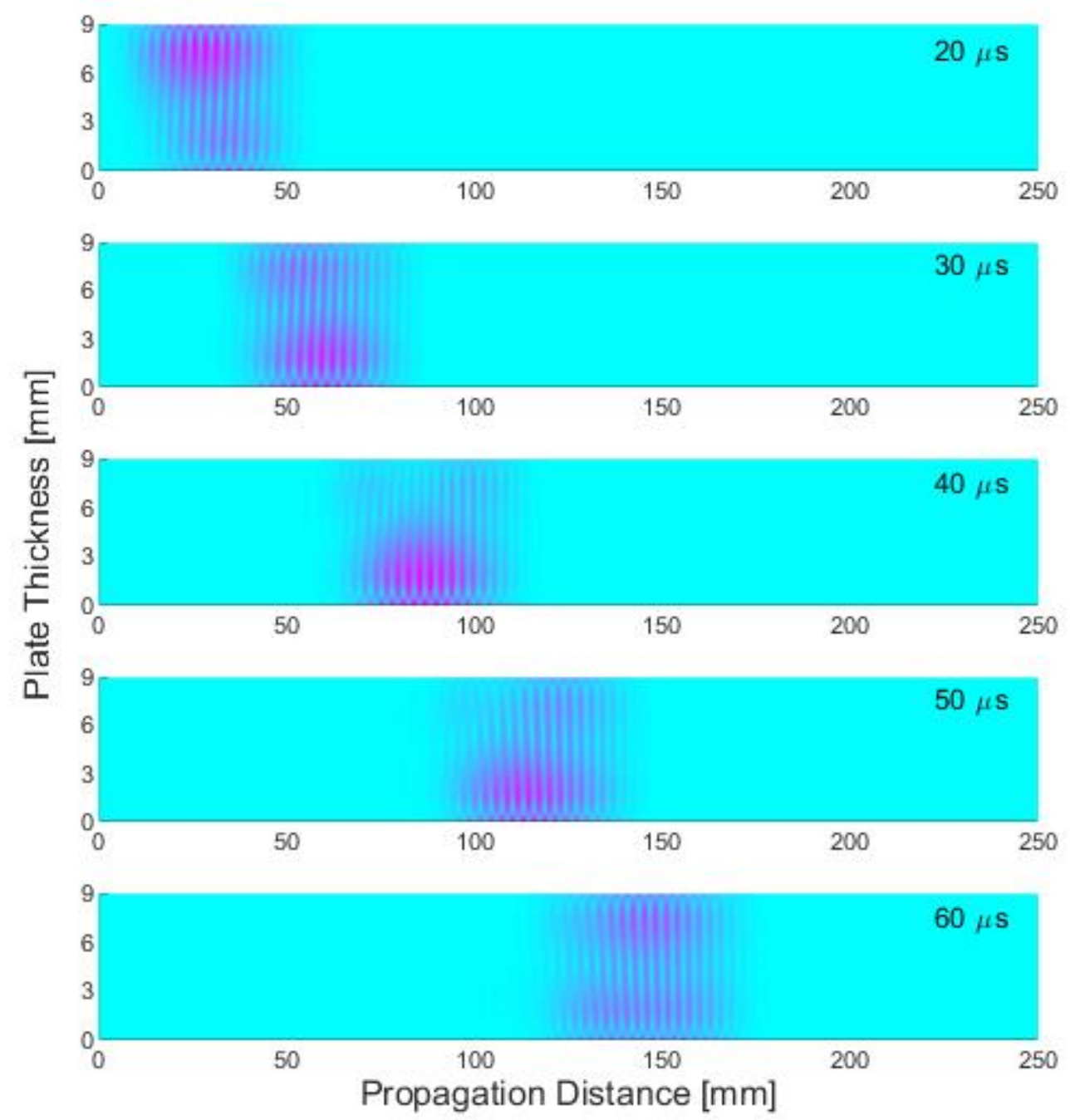

Figure 2. 2D Finite Element wave propagation simulation, showing von Mises stress at different time steps in $9 \mathrm{~mm}$ thick mild steel plate specimen $(0.5 \mathrm{MHz} ; 10$ cycles $)$.

\section{GUIDED WAVE INTERFERENCE VISUALIZATION AND PREDICTION}

The wave propagation along a $9 \mathrm{~mm}$ thick steel plate was visualized from the 2D FE simulation, with time snapshots of the von Mises stress field shown in Fig. 2 . As the guided wave modes $\left(\mathrm{A}_{0}\right.$ and $\mathrm{S}_{0}$ Lamb modes) propagate along the plate, energy is transferred gradually from the top (excitation) part to the bottom part and then back towards the top. This beating effect occurs due the slight difference in wavenumbers between the fundamental $\mathrm{A}_{0}$ and $\mathrm{S}_{0}$ modes at the chosen frequencythickness product (Fig. 1). As the wave propagates along the plate, the different phase velocities of the $A_{0}$ and $S_{0}$ modes lead to a gradually increasing difference in phase from the initial in-phase excitation with an associated shift in energy and lower amplitude at the top surface.

Figure 3 shows the recorded time traces on the top and bottom surfaces. The decrease in amplitude at $90 \mathrm{~mm}$ propagation distance and consecutive increase at $150 \mathrm{~mm}$ propagation distance at the top surface can be observed. This is matched by an increase and consecutive decrease of the wave pulse amplitudes recorded on the bottom surface. As the beating effect is frequency-dependent and the excited 10-cycle pulse has a frequency content from approximately $0.4 \mathrm{MHz}-0.6 \mathrm{MHz}$, the time signal amplitude does not go to zero. E.g., at $90 \mathrm{~mm}$ propagation distance on the top surface it can be observed that the time signals splits into two smaller pulses as some energy remains on the top surface. 
Top
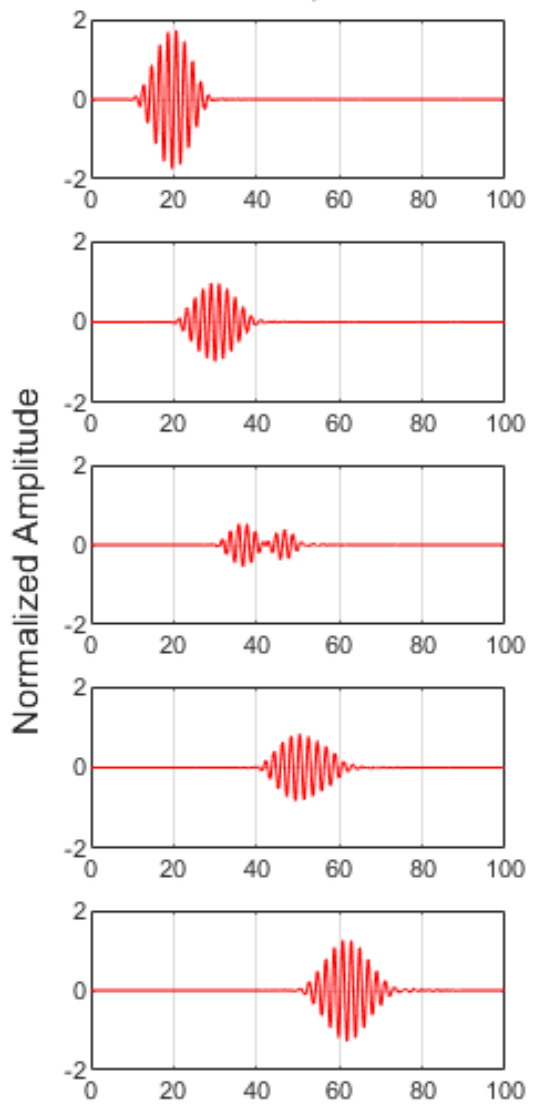

Bottom
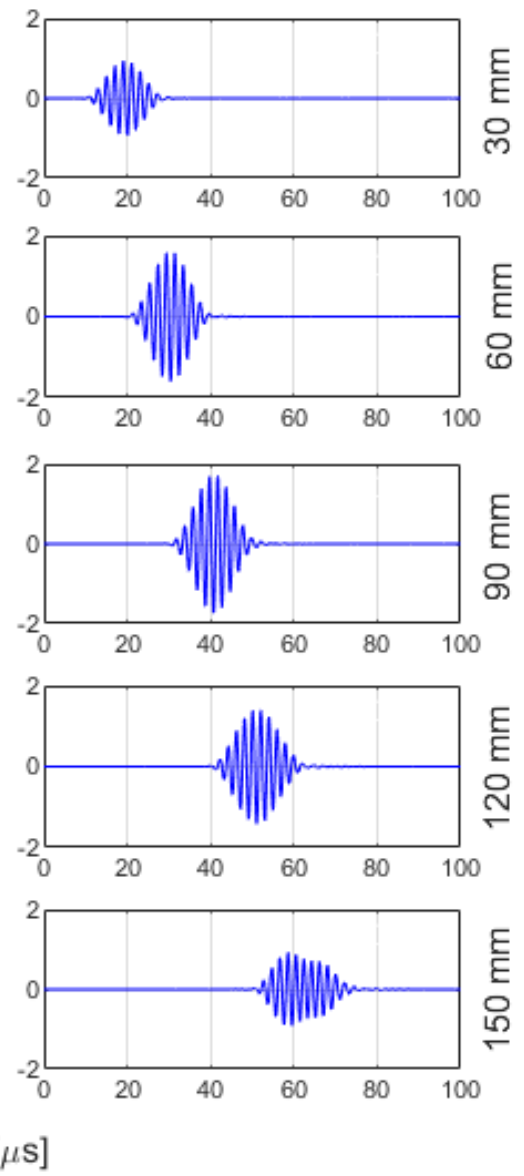

Figure 3. FE simulation of wave propagation along 2D plate specimen, displacement (out-of-plane) time traces at different locations, top and bottom surfaces; excitation frequency $0.5 \mathrm{MHz} ; 10$ cycles; $9 \mathrm{~mm}$ thick steel plate.

The signal magnitude at $0.45 \mathrm{MHz}$ was extracted using Fast Fourier Transform (FFT) and is shown along the top surface for four models with different constant plate thicknesses in Fig. 4 (left). Depending on the difference in the wavenumber of the $A_{0}$ and $S_{0}$ modes for a given plate thickness, the evaluation in the frequency domain shows a clear interference pattern with decreasing and increasing amplitude according to the beatlength.

For plate thicknesses decreasing from $11 \mathrm{~mm}$ to $9 \mathrm{~mm}$ the difference in wavenumbers of the fundamental modes increases (Fig. 1) and the beatlength decreases. The shorter beatlength can be clearly observed and was evaluated by curve fitting in Matlab, taking into account the amplitude and phase at the start of the propagation distance and the overall exponential decrease due to attenuation. Good agreement of the fitted curves can be observed in Fig 4 (left), even though the amplitudes extracted from the numerical simulation do not go to zero amplitude at the minima due to slightly unbalanced excitation of the two fundamental modes.

The beating effect depends on the frequency-thickness product, and the beatlength was extracted from FE simulations for plate thicknesses between $11 \mathrm{~mm}$ and $9 \mathrm{~mm}$ (in steps of $0.1 \mathrm{~mm}$ ). The resulting beatlength values were compared to theoretical predictions, using the calculated difference of the wavenumbers from Disperse (Fig. 1). As expected, very good agreement was found with a maximum difference of $1 \%$ (Fig. 4, right), with the small differences due to numerical dispersion and inaccuracies in the fitting procedure. For the plate thickness range of interest, a significant change of the beatlength was predicted, with a reduction in beatlength from approximately $290 \mathrm{~mm}$ to $125 \mathrm{~mm}$ for a thickness change of $2 \mathrm{~mm}$. 

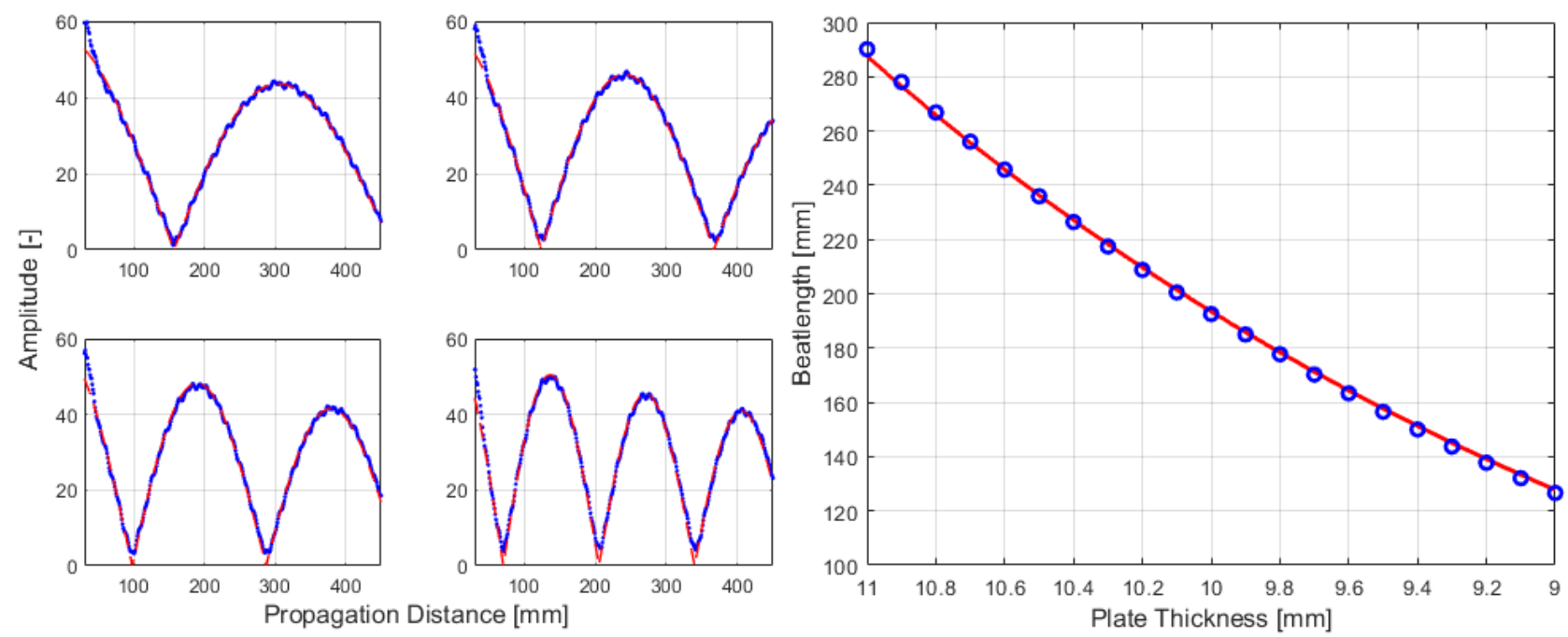

Figure 4. 2D FE simulation results; Left: amplitude of guided wave propagation, extracted at $0.45 \mathrm{MHz}$ (FFT) from time traces along top surface (blue dots: simulation amplitude; red line: exponential fit) for $11.0 \mathrm{~mm}, 10.4 \mathrm{~mm}, 9.8 \mathrm{~mm}$, $9.0 \mathrm{~mm}$ respectively; Right: beatlength at $0.45 \mathrm{MHz}$ from FE simulations (blue circles) compared to theoretical prediction (red line).

\section{EXPERIMENTS}

Mild steel (EN3B - AISI 1020) plates (length: $650 \mathrm{~mm}$; width: $100 \mathrm{~mm}$ ) were milled to an initial thickness of $11 \mathrm{~mm}$. To achieve uniform thickness reduction, one specimen was milled down in $0.2 \mathrm{~mm}$ steps to $9.4 \mathrm{~mm}$ thickness. The thickness of two specimens was reduced using accelerated corrosion for the plates partially submerged in salt water. A DC current with the plate as the anode was applied and the wave propagation measured when the thickness had reduced by approximately $0.2 \mathrm{~mm}[23]$.

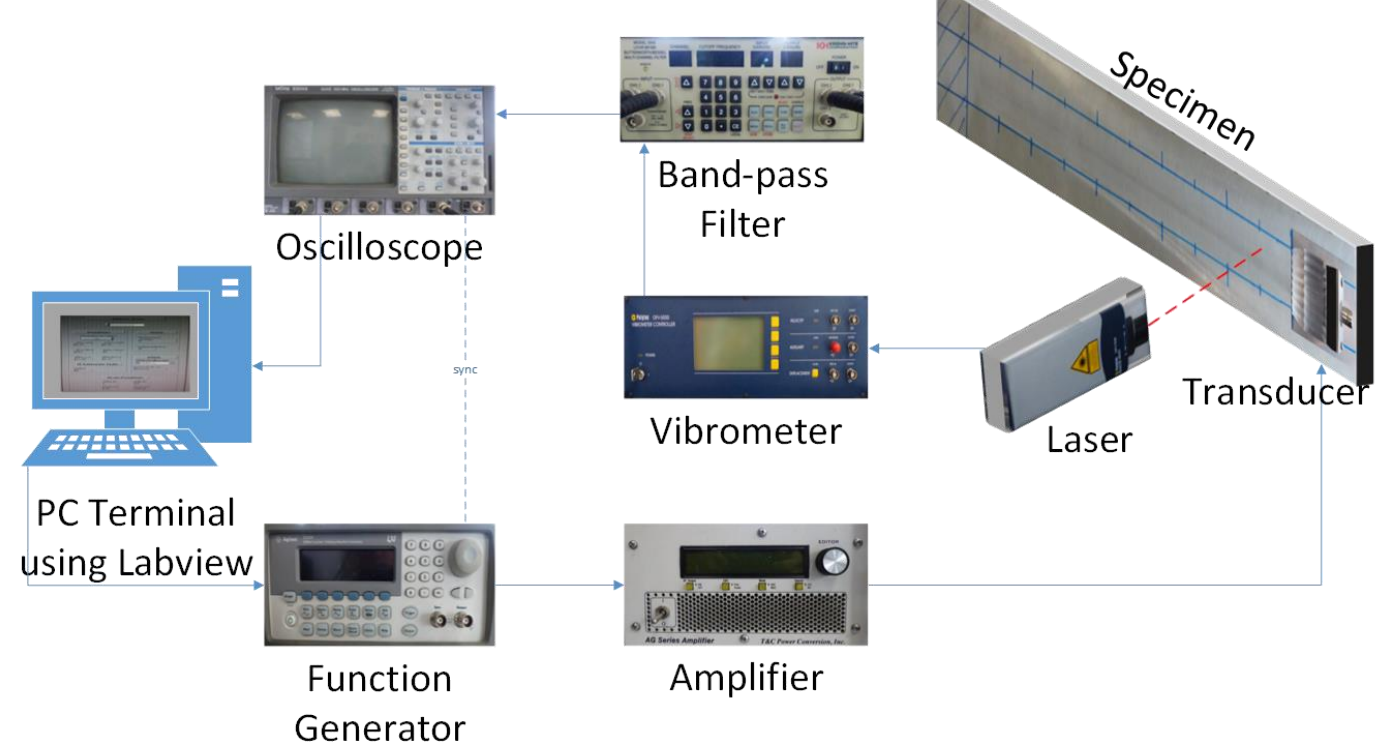

Fig. 5. Experimental setup for high frequency guided ultrasonic wave propagation measurement on steel plates [2]. 
Using an angle beam transducer (Rayleigh angle wedge for steel) with center frequency of $0.5 \mathrm{MHz}$ placed on the top surface of the plate, a 10-cycle tone burst wave pulse was excited, as in the FE simulations. The excitation signal was defined using an arbitrary function generator and amplified using a broadband power amplifier. The wave propagation along the center line of the plate specimens was measured in $1 \mathrm{~mm}$ steps over a distance of $450 \mathrm{~mm}$ using a Polytec vibrometer moved parallel to the plate on a positioning system (Fig. 5). The guided wave signal was band pass filtered $(0.4 \mathrm{MHz}-0.6 \mathrm{MHz}$ ), averaged (50 averages) and sampled using a LeCroy oscilloscope. To match the evaluation of the numerical simulations, the magnitude at $0.45 \mathrm{MHz}$ frequency was extracted using Fast Fourier Transform (FFT) in Matlab for each recorded time signal.

\section{MEASUREMENT OF THICKNESS REDUCTION}

Figure 6 shows the amplitude variation measured for the milled plate specimen and one of the corroded specimens for two different plate thicknesses, respectively. The characteristic beating pattern with a periodic decrease and increase of amplitude with propagation distance can be observed, as in the numerical simulations. More variability of the amplitude pattern than for the FE simulations can be seen, with the amplitudes not going to zero at the minima, especially for the thinner plate specimens. The small, periodic variation of the amplitude is likely due to an interference with a higher wave mode. The evaluation for the plate subjected to accelerated corrosion shows slightly larger amplitude variations, possibly due to the roughness and unevenness of the corroded surface, but the beating pattern can be clearly observed. Good overall agreement was found with the fit using the expected exponentially decreasing cosine curve, with the beatlength decreasing with decreasing plate thickness for all three specimens.

The beatlength was extracted for all measurements with thickness reductions in approximately $0.2 \mathrm{~mm}$ steps and is compared to the analytically calculated beatlength in Fig. 7. Overall good agreement and the expected significant reduction of the beatlength with plate thickness can be observed. For thicker plates corresponding to larger beatlengths, the match is not as good as for shorter beatlengths.

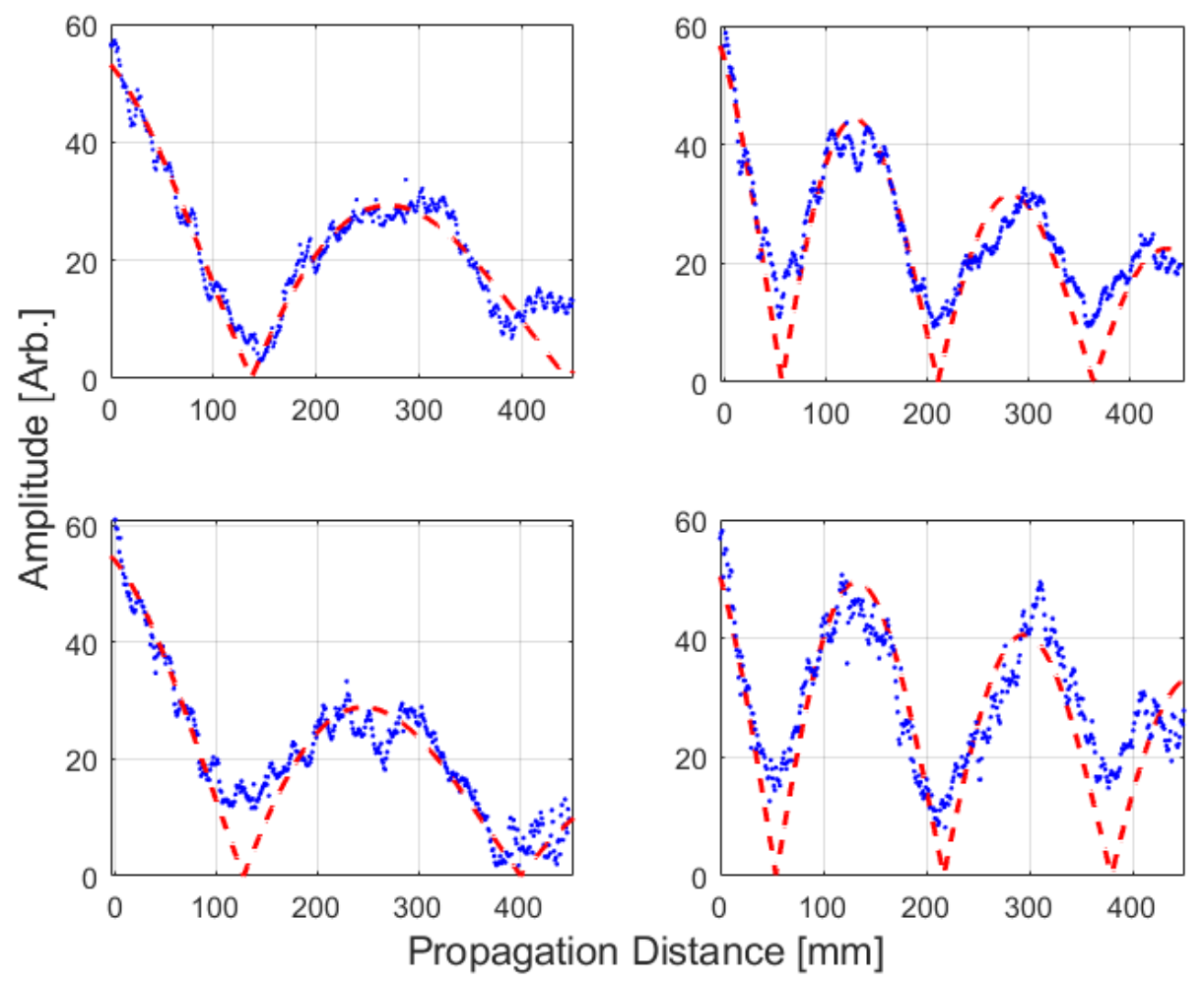

Fig. 6. Experimental amplitude variation along propagation length, FFT at $0.45 \mathrm{MHz}$ for milled specimen (top) and corroded specimen (bottom); Left: approximately $10.8 \mathrm{~mm}$ thickness; Right: approximately $9.4 \mathrm{~mm}$ thickness; blue dots: measured amplitude; red line: exponential fit. 


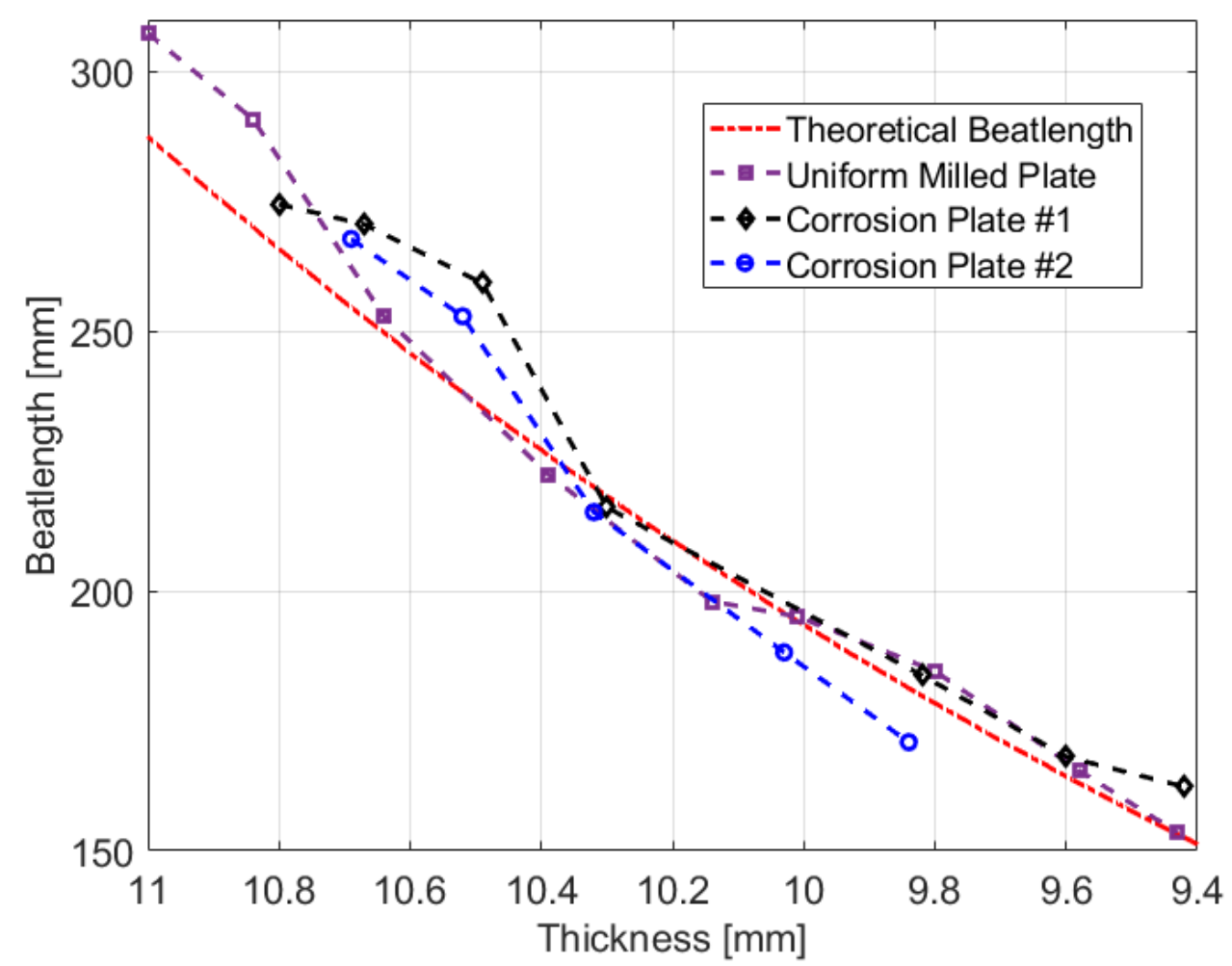

Fig. 7. Beatlength comparison of measured values (1 milled, 2 corroded specimens) against analytical prediction; $0.45 \mathrm{MHz}$ frequency evaluation.

The beatlength of $290 \mathrm{~mm}$ at $11 \mathrm{~mm}$ thickness is comparable to the measurement length of $450 \mathrm{~mm}$ and the accuracy of the fitting procedure is reduced, as only a limited number of maxima and minima lie within the propagation distance. For shorter beatlengths corresponding to thinner plates the accuracy of the fitting procedure increased, as more maxima and minima are visible within the measured propagation distance (Fig. 6). The experimental beatlength evaluation was found to be very sensitive to the plate thinning and it was estimated that the plate thickness can be quantified with approximately the thickness steps of $0.2 \mathrm{~mm}$, corresponding to about $2-3 \%$ thickness reduction.

\section{CONCLUSIONS}

Wall thickness loss due to corrosion is an important problem for a range of engineering applications, ranging from pipelines to marine structures such as offshore oil platforms. The monitoring using high frequency guided waves was investigated and the evaluation using the beatlength was found to be very sensitive to changes in the frequency-thickness product. From 2D Finite Element simulations the guided wave propagation and interference along steel plates was visualized and predicted. Excellent agreement and high sensitivity were predicted based on the evaluation of the numerical simulations in the frequency domain. Experimentally, the guided wave modes were selectively excited using Rayleigh angle wedge transducers and measured using a noncontact laser vibrometer. Good propagation distance along the plate specimens was achieved, both for a milled specimen and two specimens subjected to accelerated corrosion. The evaluation of the experimental measurements showed good agreement with the analytically predicted significant reduction of beatlength with wall thickness loss, and the sensitivity under laboratory conditions was estimated at approximately $3 \%$ thickness reduction. Further measurements should be conducted to verify the predicted sensitivity for field measurements and to check the applicability for curved specimens.

\section{ACKNOWLEDGEMENTS}

The authors would like to thank Daniel Chew for his contribution to the development of the corrosion measurement procedure and the UCL Mechanical Engineering workshop for the precision machining. 


\section{REFERENCES}

[1] Nakai, T., Matsushita, H., Yamamoto, N. and Arai, H., "Effect of pitting corrosion on local strength of hold frames of bulk carriers (1st report)," Marine Struct. 17, 403-432 (2004).

[2] Chew, D. and Fromme, P., "Monitoring of corrosion damage using high-frequency guided ultrasonic waves," Proc. SPIE 9064, 90642F (2014).

[3] Cawley, P., Cegla, F. and Stone, M., "Corrosion Monitoring Strategies - Choice Between Area and Point Measurements,” J. Nondestruct. Eval. 32, 156-163 (2013).

[4] Rose, J. L., "Standing on the shoulders of giants: An example of guided wave inspection," Mat. Eval. 60, 53-59 (2002).

[5] Hall, J.S., Fromme, P. and Michaels, J.E., "Guided Wave Damage Characterization via Minimum Variance Imaging with a Distributed Array of Ultrasonic Sensors," J. Nondestruct. Eval. 33, 299-308 (2014).

[6] Fromme, P. and Sayir, M.B., "Measurement of the scattering of a Lamb wave by a through hole in a plate," J. Acoust. Soc. Am. 111, 1165-1170 (2002).

[7] Alleyne, D.N. and Cawley, P., "The interaction of Lamb waves with defects," IEEE Trans. Ultrason. Ferroelectr. Freq. Control 39, 381-397 (1992).

[8] Huthwaite, P., Ribichini, R., Cawley, P. and Lowe, M.J.S., "Mode Selection for Corrosion Detection in Pipes and Vessels via Guided Wave Tomography," IEEE Trans. Ultrason. Ferroelectr. Freq. Control 60, 1165-1177 (2013).

[9] Nagy, P.B., Simonetti, F. and Instanes, G., "Corrosion and erosion monitoring in plates and pipes using constant group velocity Lamb wave inspection”, Ultrasonics 54, 1832-1841 (2014).

[10] Fromme, P., "Health Monitoring of Plate Structures using Guided Waves," Proc. SPIE 6935, 69350W (2008).

[11] Fromme, P., Wilcox, P., Lowe, M. and Cawley, P., "On the development and testing of a guided ultrasonic wave array for structural integrity monitoring," IEEE Trans. Ultrason. Ferroelectr. Freq. Control 53, 777-785 (2006).

[12] Masserey, B. and Fromme, P., "Fatigue Crack Growth Monitoring using High Frequency Guided Waves," Struct. Health Monit. 12, 484-493 (2013).

[13] Howard, R. and Cegla, F., "On the probability of detecting wall thinning defects with dispersive circumferential guided waves," NDT \& E Int. 86, 73-82 (2017).

[14] Terrien, N., Osmont, D., Royer, D., Lepoutre, F. and Déom, A., "A combined finite element and modal decomposition method to study the interaction of Lamb modes with micro-defects," Ultrasonics 46, 47-78 (2007).

[15] Greve, D.W., Zheng P. and Oppenheim I.J., "The transition from Lamb waves to longitudinal waves in plates," Smart Mater. Struct. 17, 035029 (2008).

[16] Ratnam, D., Balasubramaniam, K. and Maxfield, B.W., "Generation and Detection of Higher-Order Mode Clusters of Guided Waves (HOMC-GW) Using Meander-Coil EMATs,” IEEE Trans. Ultrason. Ferroelectr. Freq. Control 59, 727-737 (2012).

[17] Burch, S.F., Collett, N.J., Terpstra, S. and Hoekstra, M.V., "M-skip: A quantitative technique for the measurement of wall loss in inaccessible components," Insight 49, 190-194 (2007).

[18] Khalili, P. and Cawley, P., "The choice of ultrasonic inspection method for the detection of corrosion at inaccessible locations," NDT \& E Int. 99, 80-92 (2018).

[19] Masserey, B., Raemy, C. and Fromme, P., "High-frequency guided ultrasonic waves for hidden defect detection in multi-layered aircraft structures," Ultrasonics 54, 1720-1728 (2014).

[20] Masserey, B. and Fromme, P., "In-Situ Monitoring of Fatigue Crack Growth using High Frequency Guided Waves," NDT\&E Int. 71, 1-7 (2015).

[21] Viktorov, I.A., "Rayleigh and Lamb Waves," Plenum Press, New York, 93-96 (1967).

[22] Masserey, B. and Fromme, P., "On the reflection of coupled Rayleigh-like waves at surface defects in plates," J. Acoust. Soc. Am. 123, 88-98 (2008).

[23] Chew, D., Masserey, B. and Fromme, P., "High-Frequency Guided Waves for Corrosion Thickness Loss Monitoring," ASME J. Nondestructive Evaluation, Diagnostics, Prognostics of Engineering Systems 4, 011007 (2021).

[24] Ti, B.W., O'Brien, W.D. and Harris, J.G., "Measurements of coupled Rayleigh wave propagation in an elastic plate," J. Acoust. Soc. Am. 102, 1528-1531 (1997).

[25] Auld, B.A., "Acoustic Fields and Waves in Solids," Wiley, New York, Vol. 2, 93-94 (1973). 